the place of interment both of Charles White himself and of bis youngest son.

It may be that now and then White yielded to a temptation to which men of predominant intellect and force of character are specially prone, the temptation to let others see and feel the superiority of which they themselves are conscious. Stories have come down to us which show that he did occasionally in his behaviour towards his professional brethren carry matters with rather a high hand, but certainly nothing could exceed the admiration expressed for his character and work both in Manchester and elsewhere by those who being leaders of the profession to which he belonged were best qualified to judge. It is on record that he possessed a clear and pleasant voice and that he was an impressive and inspiring teacher. His painstaking manner in the investigation of a case and his promptitude and firmness in coming to a decision gave his patients unlimited confidence in him. He was a genial companion ever ready to gather as well as to impart information, and his conversa. tion is said to have abounded with entertaining anecdotes. He was a man of whom Manchester will always feel proud and in taking leave of him to-day we can adopt with very slight modification the graceful words addressed to him towards the close of his life by his old friend Dr. Rigby of Norwich, ${ }^{17}$ and, after the lapse of nearly 100 years, can still truthfully say "that time has in no degree lessened the sense of our obligation to him, nor can it diminish the great respect that has ever been entertained for his high character as a surgeon, as an author, and as a man."

\section{THE QUANTITATIVE AND QUALITATIVE RELATIONS OF TOXIN AND ANTITOXIN.}

BY E. F. BASHFORD, M.D. EDIN,

- ENERAL SUPERINTENDENT, CANCER RESEARCH FUND; GROCERS' RESEARCH SCHOLAR, ETC.

Pakt I. - Some Conclusions DRawn From the Abolition of the Fatal action of a Lethal Dose of TOXIN BY ANTITOXIN, AND THE ABSENCE OF THIS Abolition IF the HundRedfold Multiples BE TAKEN.

THE following paper has only an indirect bearing upon the routine methods of testing and standardising diphtheritic and other antitoxic sera. These methods fulfil the requirements of testing and are not, from the practical aspect, subject to the following criticism of the hypotheses which have been advanced in explanation of phenomena met with in the course of following them out.

It is usually stated that the quantity of antitoxic serum necessary to render no longer lethal a fatal dose of diphtheria toxin bouillon neutralises the latter. It is one of the purposes of this note to emphasise the limitations within which this method of stating the neutralisation process is true and to point out the important bearing of these limitations on some hypothetical explanations of the apparent nonfulfilment of what is known as the law of multiples when solutions of toxin and antitoxin react with one another in different volumes. The term "neutralisation" is indiscriminately applied both to the reaction between toxin and antitoxin, and to its consequence-viz., diminution or complete loss of the full toxicity of a quantity of toxin. Although intimately related, the two are distinct phenomena. It is unfortunate that the distinction between them is not more generally made. A chemically neutral mixture of toxin and antitoxin will be a physiologically neutral one. What is usually called a physiologically neutral mixture is not necessarily a chemically neutral one ; the former takes no account of molecular quantities. Much confusion is obviated by keeping these distinctions in mind. It must also be remembered that cells or animals, as indicators of the physiological neutrality of a mixture of toxin and antitoxin, do not permit of the sharp definition attainable in determining chemical neutrality, and also, as with some chemical indicators-e.g., turmeric which only gives evidence of free alkali-the physiological indicators only respond to one of the factors in the

17 See the dedication to the fifth edition of his famous monograph on uterine hæmorrhage. reacting mixture-viz., the toxin-if the latter be present in sufficient excess to produce illness. In dealing with toxins and antitoxins we have no direct indications of chemical neutrality.

One of the fundamental assumptions of some very prevalent views on the nature of immunity is that 100 times the quantity of antitoxic serum needful to render non-lethal the fatal dose of toxin should render 100 times the fatal dose innocuous. ${ }^{1}$ The requirement is practically never fulfilled and it is customary to point out that its fulfilment can alone be possible if the toxin bouillon contains only a single factor capable of reaction with the antitoxin. Conversely the nonfulfilment of the requirement is held to be an argument in favour of the assumption that more than one factor is able to react with the antitoxin. Because the hundredfold multiple of antitoxic serum is not found to render non-lethal so much as 100 times the fatal dose of toxin bouillon it is stated that there must be contained in the latter other factors than the toxin able to neutralise antitoxin, and this assumption requires the further hypothesis that any factors present cannot be possessed of the characteristic toxic action of the toxin, but they must nevertheless still be possessed of the latter's power to neutralise the specific antitoxin. It is only fair to point out that further reasons are also adduced in support of the view that non-toxic products present in diphtheria toxin bouillon may still be antitoxin-neutralising bodies, and I may add that because guinea-pigs frequently die subacutely with paralytic symptoms after receiving a mixture of diphtheria toxin and antitoxin it has been assumed that this is further evidence of the existence of a relatively non-toxic modification of the toxin. Into the question of the relation of the toxic power of a toxin to its antitoxin-neutralising power I do not now wish to enter.

In the first place $I$ wish to discuss why it is not justifiable to require that the hundredfold multiple of the quantity of antitoxin that is able to render non-letbal a lethal dose of toxin should render 100 such lethal doses innocuous.

1. In experiments performed some time ago in Berlin I found, after preliminary determinations, the dose of a diphtheria toxin bouillon which killed five guinea-pigs each of 250 grammes weight between the fourth and the fifth day to be 0.0075 cubic centimetre (contained in 2.25 cubic centimetres of 1 in 300 dilution). The dose which did nos kill any of another similar series till between the fifth and sixth day was 0006 cubic centimetre (contained in $\mathbf{1 . 8}$ cubic centimetres of 1 in 300 dilution). In each case the quantity was made up to four cubic centimetres with 0.86 per cent. of saline solution. The syringe was washed out with saline solution and the washings were also injected. The injections were made over the sternal region. Previous attempts to secure two such series as above had given wider margins of difference between the doses of the two series and also between the intervals at which the fatal issue occurred. When the difference between the doses of the two series specified amounted to one-fifth of the determined lethal dose $(0.0075$ cubic centimetre) it was possible to obtain two series distinguished from one another by one day's interval in the manifestation of the lethal issue. The attainment of this result was probably exceptional because I have not again been able to obtain two doses one of which always killed on the fourth and the other on the fifth day. The smaller dose was as apt to kill on the fourth as the larger was to kill on the fifth day. The two successful series, however, were sufficient to show that the diminution by one. fifth of its quantity sufficed to prevent the fatal dose from fulfilling the requirement that it should kill within four days. In a further series of experiments it was determined that a quantity somewhere between onetwelfth and one-twentieth of the above lethal dose did not manifest itself in any symptoms-i.e., the animals did not lose weight. I did not attempt to define the largest portion of the lethal dose which could be borne without manifest illness. It was sufficient to have obtained a measurable quantity thus characterised.

2. When a series of mixtures of toxin bouillon and antitoxic serum was made, so that in the same bulk of fluid the determined lethal dose was always contained, together with varying amounts of antitoxic serum, that dose of serum which rendered the lethal dose non-lethal within the time limit of four days was determined. Very little serum sufficed

1 The fatal dose more particularly referred to is the dose of diphtheria toxin bouillon which kills a 250 -gramme guinea-pig within four days, but on this special case very far-reaching general statements are based. 
to postpone death (which was still acute) to the fifth or the sixth day, The quantities effecting this postponement were only fractions of the total amount of antitoxin necessary to prevent a lethal issue within four days and still smaller fractions of the amount necessary to prevent absolutely a fatal termination. I do not wish to suppose that these fractions of serum were equivalent to the previously determined diminution by one-fifth in the lethal dose of the bouillon; the postponement of the lethal issue may possibly have been effected by the abolition of far smaller fractions of the full toxic power of the lethal dose. With a greater proportion of antitoxic serum added and the apparent absence of all symptoms. there was probably not less than from one-twelfth to one-twentieth of the toxin dose "free," as determined in the section above. The amount "free" may here have been considerably above this fraction. There was no means by which it could be determined that these last fractions of the full toxic power had been rendered completely innocuous, although by the arbitrary addition of excess of antitoxin this would easily have been effected. It is needless to enter into further de tails of " neutralisation" series, such as are familiar to all pathologists and easily accessible in many papers. In the reaction between a toxic bouillon and an antitoxic serum there is, therefore, a phase of which the boundaries cannot be accurately defined. If one does not assume that a molecule of the one reacts with a molecule of the other, there is, on the one hand, the possibility of the presence in the mixture of a measurable fraction of unneutralised toxicity, and on the other hand, of unneutralised-i.e., excessive-antitoxicity. Just as in section 1 the line of demarcation is indefinite. A series of doubtful doses intervenes between the certainly lethal and the certainly non-lethal. It is pure hypothesis to suppose that toxin and antitoxin can only react in one fixed proportion. It is further to be noted that it has become the fashion to estimate all these reactions on the basis of a lethal dose which kills within four days. Whereas the four-day limitation allows of greater rapidity in arriving at comparative results, it does not give greater but less accuracy in determining certainly lethal from the certainly non-lethal doses. The zone between all the certainly lethal and all the certainly non-lethal doses may be a narrower one and have more definite limitations if the absolute minimal lethal dose be determined. When one is dealing with the absolute minimal lethal dose and the abolition of the lethal action by means of antitoxin, one has at any rate a definite issue to look for in obtaining comparative results. If the period of observation be limited, however, to a period of four days within which the issue must be lethal or non-lethal this definiteness is lost. ${ }^{3}$ No attention is paid to the symptoms of illness, which must vary from nil to any degree of seriousness in accordance with the size of the fraction of unneutralised toxicity. All degrees of neutralisation extending over the wide zone lying between excessive addition of antitoxin and very limited neutralisation of toxicity are thus classed in the same category as "neutralised" and no attempt is made to secure, nor would it be possible to obtain, the same margin of error in all the observations com. pared. In making the determinations all that is sought for is such an addition of antitoxin that death does not occur at all, or at any rate not within four days, and obviously these

2 To designate this fraction of toxin "free" will only be justifiable, if after completion of the reaction between toxin and antitoxin as such the product of the reaction be indifferent to any remaining toxin. It will subsequently be shown that there is some reason for believing that the product of the reaction is not indifferent in this way, but, on the contrary, liable to enter into a combination with a higher proportion of contrary, liable to enter into a combination with a higher proportion of bined thus still further reducing the amount of the free "or uncomtoxin in such a mixture "free" also postulates the manner in which toxin in such a mixture tree also postulates the manner in which reacts with a corresponding molecule of the other, quantitative excess reacts with a correspon

of either being possible.

I do not wish to call in question the utility of this time limitation in determining the occurrence or abolition of a lethal issue. For the practical purposes of testing and standardising antitoxic sera such a time limitation is essential. The method has been productive of advances in knowledge which would have been unattainable without it. Where the fatal termination occurs after three or fyur weeks comparative results are difficult or scarcely possible of attainment, especially with bodies so liable to change in potency as toxins and antitoxins. The time limitation allows of the more rapid accumulation of experimental results and, therefore, of the more quick attainment of the goal in an investigation. In generalising from such experiments, however, in the conclusions the introduction of the time limit at the outset is almost always forgotten, as is also the fact that the time limitation is a mere experimental convenience as above explained. results will be obtained by very different quantities of anti. toxin. This procedure, while eminently serviceable for the practical purposes of standardising antitoxin, is not sufficiently accurate for the retical purposes. The wideness of the limitations of what is regarded as "neutralisation" is, I consider, therefore quite sufficient to account for the 100 antitoxin equivalents corresponding " to 16 and in another extreme instance to 136 lethal guinea-pig doses," according as there had been physiologically really " nnder- or overneutralisation" respectively of the lethal dose multiplied. In experiments of this nature the facts ascertained have reference only to physiological neutralisation and it is not correct to interpret these as having a direct and equal bearing upon chemical neutralisation.

3 From what has been said abore it is obvious that to render non-letbal within four days a dose of toxin bouillon, proved to be lethal within that period, it is necessary to abolish the potency of only a fraction of the total toxicity, to reduce the latter, indeed, to the equivalent of that of a dose fatal in five days as noted in section 1 . Similarly, to render non-lethal the absolute minimal lethal dose, it is only needful to abolish such a fraction of the full toxicity as shall reduce this latter to the non-lethal dose still sufficiently large to cause illness-i.e., complete abolition of the whole effect is not required. Fraser has emphasised this for snake venom : "While 0.35 cubic centimetre of antivenene per kilogramme is required to prevent death from twice the minimum lethal dose of venom, the minute quantity of the $\frac{1}{260}$ th of a cubic centimetre, or nearly 1000 times less $(0.0004$ as compared with 035 ), is sufficient to prevent death from a little more than the minimal lethal dose of venom ; ...... if this dose be the actual minimum lethal, the rendering inert of any portion of it, however minute, will prevent the remainder from causing death." Kanthack and Cobbett have also drawn attention to the same phenomenon. Cobbett obviated the error involved in taking the simple lethal dose as unity, by taking two lethal doses for the starting point, and then determining the ratios for $2,11,101$, and 1001 lethal doses. I take the liberty of directing special attention to Cobbett's experiments in this connexion, for they seem to have been overlooked. In the pharmacological antagonism of drugs-viz., in the abolition of the lethal action-a similar phenomenon is met with. In the abolition of the lethal action of one and a half minimal letbal doses of morphine this is effected in the accompanying table both by 0.07 milligramme

Abolition of Fatal Action of One and a Half Minimal Lethal Doses of Morphine Tartrate.

\begin{tabular}{|c|c|c|c|c|c|c|}
\hline \multirow{2}{*}{ 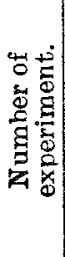 } & \multirow{2}{*}{ 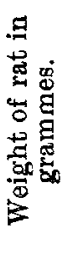 } & \multicolumn{2}{|c|}{$\begin{array}{c}\text { Dose of atropine } \\
\text { sulphate in } \\
\text { milligrammes. }\end{array}$} & \multicolumn{2}{|c|}{$\begin{array}{c}\text { Dose of mor- } \\
\text { phine tartrate } \\
\text { in milli- } \\
\text { grammes. }\end{array}$} & \multirow{2}{*}{ Result. } \\
\hline & & 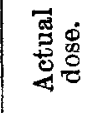 & 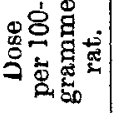 & 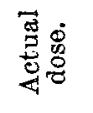 & 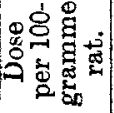 & \\
\hline 55 & 204 & 0.0412 & 0.02 & $173 \cdot 0$ & $85 \cdot 0$ & + after 4 hours. \\
\hline 56 & 140 & 0.06 & 0.05 & $119 \cdot 0$ & , & + before 17 hours. \\
\hline 57 & 187 & 0.1125 & 0.06 & $159 \cdot 0$ &, & + after 6 hours. \\
\hline 58 & 142 & 0.1 & 0.07 & $128 \cdot 7$ & ", & 0 on 6 th day. \\
\hline 59 & 184 & 0.1372 & 0.075 & $156 \cdot 2$ & ", & + after 6 hours. \\
\hline 60 & 210 & $1 \cdot 525$ & $0 \cdot 25$ & $178 \cdot 0$ & , & 0 after 5 days. \\
\hline 61 & 210 & $1 \cdot 0$ & 0.5 & $178 \cdot 0$ & ", & 0 after $4-5$ days. \\
\hline 62 & 195 & $1 \cdot 0$ & 0.6 & $165 \cdot 0$ & ," & 0 after $3-4$ days. \\
\hline 63 & 325 & $2 \cdot 4$ & 0.75 & $278 \cdot 0$ & , & $+\left(^{*}\right)$ after 6 days. \\
\hline 64 & 134 & $1 \cdot 2$ & $0 \cdot 9$ & 1150 & ", & 0 after $5-6$ days. \\
\hline 65 & 147 & $6 \cdot 0$ & $4 \cdot 081$ & $125 \cdot 0$ & ", & 0 after 3 days. \\
\hline 66 & 156 & $7 \cdot 0$ & $4 \cdot 5$ & $133 \cdot 0$ & ", & 0 after $3-4$ days \\
\hline 67 & 138 & $6 \cdot 25$ & $4 \cdot 6$ & $117 \cdot 0$ & ," & + before 17 hours. \\
\hline 68 & 124 & $6 \cdot 0$ & $4 \cdot 84$ & $105 \cdot 0$ &, & + before 20 hours. \\
\hline 69 & 155 & $7 \cdot 75$ & $5 \cdot 0$ & $132 \cdot 0$ & ", & + before 22 hours. \\
\hline 70 & 124 & $7 \cdot 0$ & $5 \cdot 66$ & $105 \cdot 0$ & , & + before 17 hours. \\
\hline
\end{tabular}

+ means deard; 0 means alive. These experiments are quoted from an extensive investigation of this subject. Vide Archives Internationales de Pharmacodynamie, vol. viii.. 1901 .

${ }^{*}$ ) This rat was exceptionally fat and heavy; in consequeuce of the excessive amount of fat the dose must be regarded as considerably higher than 85.0 milligrammes per 100 grammes.

4 Transactions of the Jenner Institute of Preventive Medicine 1899 , p. 6. 
and 4.5 milligrammes of atropine. The latter of these doses must certainly have exercised an influence in excess of that attained by the former dose, and yet it did not abolish all 'the effects of the dose of morphine, nor for the matter of that was any dose of atropine able to effect complete abolition of the action of the morphine.

The nature of pharmacological antagonism precluded abolition of the lethal effect by a still larger dose. Pharmacological antagonism is certainly not due to direct interaction of the two active agents in question and I only quote the matter here in support of the general contention that between abolition of lethal action and complete abolition of all action (or in the case of toxin and antitoxin of complete physiological and chemical neutralisation) there is a wide margin. Obviously, therefore, to state that a certain quantity of antitoxic serum neutralises the lethal dose is a careless way of saying that it abolishes the lethal action. To require that the hundredfold multiple of the quantity of an antitoxic serum which renders non-lethal a dose of toxin shall neutralise physiologically 100 similar doses is erroneous and it is wrong to apply the data to the problems of chemical neutralisation. If it be found that instead of the action of the 100 doses only that of 60 , say, is accounted for, this fact of itself does not justify the adoption of an explanation which calls into existence in the toxin bouillon some nontoxic but antitoxin-neutralising substance-i.e., a body physiologically inert but chemically active. It is only legitimate to require that the hundredfold multiple of the quantity of serum shall account for the action of 100 times the toxic fraction abolished in the case of the simple dose of the toxin bouillon-i.e., provided the larger masses acting and their products are indifferent to the reaction, which in all probability they are not. In making comparisons for theoretical purposes it is not justifiable to assume that a constant quantity of a specific antitoxic serum must neutralise the same multiples of the lethal doses of different toxin bouillons of which the lethal doses are contained in different volumes ; for practical purposes this variation in the volumes containing a lethal dose matters little.

4. Some of the points raised in the foregoing in regard to deductions based on what occurs for a simple dose and its multiples may be illustrated by a case in which there can be no question of a non-toxic yet antitoxin-neutralising modication of a toxin, nor of an antitoxin. If corrosive sublimate dissolved in 0.86 per cent saline solution is allowed to act on one cubic centimetre of defibrinated rabbit's blood in a 5 per cent. suspension the erythrocytes will be laked after an interval of from four to six hours at $38^{\circ} \mathrm{C}$., as follows :-

Corrosive sublimate, 1 in $10,000,1.0$ c.c. ...... Complete laking.

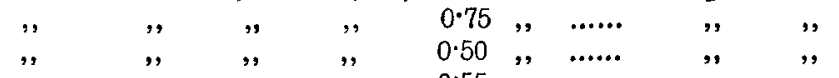

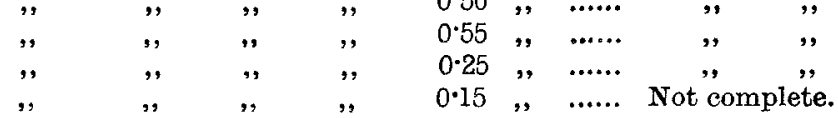

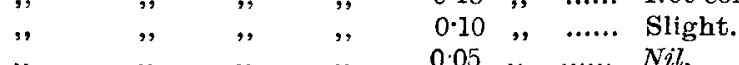

o all the test-tubes saline solution is added to raise the bulk to 2 c.c. The limits of the action vary in different specimens of defibrinated blood, probably on account, not so much of varying susceptibility as of variation in the number of erythrocytes in one cubic centimetre of the suspension due to the different extents to which they are removed during the process of defibrination. It is better to obtain more accurate determinations by using higher dilutions of corrosive sublimate and then to choose a dilution which allows of all the stages being included within a convenient compass.

In the above series 025 cubic centimetre is the lowest dose which has effected complete laking. In another series of tubes, under the same conditions of experiment, that quantity of rabbit's serum (or any other serum apparently indifferent to rabbits' erythrocytes) is determined which added simultaneously with 0.25 cubic centimetre of the corrosive sublimate solution abolishes ${ }^{5}$ the effect of the latter.

$$
\begin{aligned}
& \text { Corrosive sublimate } \\
& 1 \text { in } 10,000 \\
& 0.25 \text { c.c. } \\
& \text { Serum. } \\
& 0.55 \text { c.c. ......... } \\
& 0.30 \text {, , } \\
& 0.15 \text {, } \\
& 0 \cdot 20 \text {," } \\
& 0.15, \\
& \text { …...... } \\
& \text {.............. } \\
& \text {............ } \\
& \text { complete laking. } \\
& \text {......... } 0.10 \\
& \text { Result. } \\
& 0 \\
& 0 \\
& 0
\end{aligned}
$$

5 This is not an antitoxic action on the part of the serum, but

\begin{tabular}{|c|c|c|c|}
\hline $\begin{array}{l}\text { Corrosive sub- } \\
\text { limate (c.c.). }\end{array}$ & Result. & $\begin{array}{l}\text { Corrosive sub- } \\
\text { limate (c.c.). }\end{array}$ & Result. \\
\hline $2 \cdot 50$ & Complete laking. & 1.90 & Incomplete. \\
\hline $2 \cdot 35$ &, & $1 \cdot 85$ & Slight. \\
\hline $2 \cdot 25$ & , & $1 \cdot 80$ & ? trace. \\
\hline $2 \cdot 20$ & , & $1 \cdot 75$ & 0 \\
\hline $2 \cdot 15$ & " & $1 \cdot 70$ & 0 \\
\hline $2: 10$ & , & $1 \cdot 65$ & 0 \\
\hline $2 \cdot 05$ & , & $1 \cdot 60$ & 0 \\
\hline $2 \cdot 0$ & $"$, & 1.55 & 0 \\
\hline 1.95 & ? not complete. & & \\
\hline
\end{tabular}
analogous to certain other reactions which in annther paper I have designated as "pseudo-antitoxic," because they simulate the toxinneutralising action of an antitoxin.
Here it is only after much experimentation that the results can be obtained in this convenient compactness.

From these two series of experiments we have obtained the information that 0.25 cubic centimetre of the corrosive sublimate solution effects complete laking, that 005 cubic centimetre has no effect, that $0 \cdot 2$ cubic centimetre of serum abolishes the effect of 0.25 cubic centimetre of the sublimate solution, and that therefore in the mixture containing 0.25 cubic centimetre of sublimate and 0.2 cubic centimetre of serum there could not have been free to act more than 0.05 cubic centimetre of sublimate solution. If an experiment be now performed with the tenfold quartity of serum we shall see if this quantity be able to abolish the effect of ten times as much corrosive sublimate. The quantity of serum is two cubic centimetres and we add corrosive sublimate in the following quantities :-

There is difficulty in determining the exact limit where complete laking and no laking are present. We may take these limits, however, as being with two cubic centimetres and 1.75 cubic centimetres respectively. The difference between these two quantities is 0.25 cubic centimetre-i.e., the simple laking dose. The tenfold multiple of serum has "neutralised" not the tenfold quantity of sublimate but only somewhere between seven and eight times of what might have been expected, if one were to require here what is required in similar circumstances in the case of multiples of toxin and antitoxic sera. Here two or three complete doses of sublimate less than the tenfold quantity have not been neutralised and it is certainly impossible to assume that there is present in the corrosive sublimate solution a body derived from it without the laking action of corrosive sublimate, but with the power of the latter to enter into reaction with the albumins contained in the serum added as the "protective" agent in this case. The explanation, as shown by the following considerations, is the simple one that in the tenfold multiples the fraction not reacted on by the serum in the case of the simple dose has become augmented into a potent quantity equal at least to two simple laking doses. Of the solution of sublimate 0.25 cubic centimetre effects complete laking while 0.05 cubic centimetre is without evident effect. The addition of 02 cubic centimetre of serum abolishes the effect of 025 cubic centimetre of sublimate solution-i.e., of the 0.25 cubic centimetre not more than 005 cubic centimetre is free and not less than 0.2 cubic centimetre has been neutralised. If 02 cubic centimetre $=x$ then $0.25-x=005=20$ per cent.; if the tenfold multiple be taken then $25-10 x=05$, or two simple laking doses free. With ten multiples of the dose of sub-
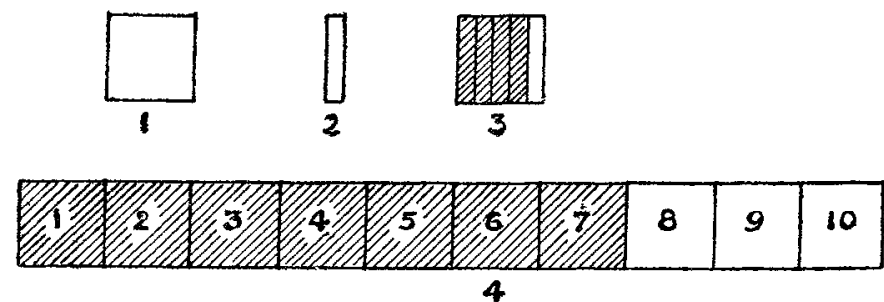

Diagrammatic representation of the results of experiments with corrosive sublimate. 1, Minimal dose taken as unity. 2, Largest dose producing no evident action. 3, Shaded area $=$ abolition of action of minimal dose; white area $=$ fraction of dose still potent. 4, Result of taking tenfold multiples : shaded area action abolished; unshaded area $=$ augmented extent of portion not abolished by serum.

limate the quantity unneutralised has become a multiple of this simple dose-i.e., a dose which was harmless has been multiplied so as to become harmful. It is therefore needless to assume that something else than the corrosive sublimate per se has used up the serum added. 
The erythrocytes are not delicate indicators enough to permit of a "neutralisation" of this nature without the introduction of an experimental error of more than one complete dose in a tenfold multiple. In the "neutralisation" the difference between the tenfold "neutralisation" required and that obtained was nearer three doses than twothe action only of seven and not of eight simple doses was completely abolished. This shows that with the hundredfold multiples there would have been a difference of from 20 to 30 doses between the neutralisation point found and that required by assuming complete "neutralisation" of the single dose to have been effected by the quantity of serum which sufficed to abolish its action. I doubt if anyone would venture to suggest that the explanation was to be found in the presence in the sublimate solution of bodies analogous to those nowadays generally believed to be responsible for the similar difference in the case of toxin and antitoxin.

5 . Guinea-pigs are not such good indicators of the presence of free diphtheria toxin as rabbits' erythrocytes are of corrosive sublimate. Earlier in this note I have mentioned that one twelfth to one-twentieth of the lethal dose of diphtheria toxin bouillon was certainly borne without any symptoms, but a much larger dose-one-sixth, one-fifth, or even onefourth-may frequently be followed by little or no sign of illness. In a "neutralisation" experiment, where the presence or the absence of a fatal issue is the criterion of "neutralisation," any lesser reaction would almost certainly be overlooked or regarded merely as transitory oedema, loss of weight, \&c., even if prominent enough to attract so much notice. When, on taking the hundredfold quantity of antitoxic serum, it is found that ten, 20 , or 30 doses less than the hundred doses of toxin expected are "neutralised," it is not correct to interpret this falling short solely on the hypothesis that in the diphtheria toxin bouillon there is present a derivative of the toxin which is not toxic but yet possessed of antitoxin-neutralising powers. Some proportion of this shortcoming is represented by the difference between the hundredth multiple of the fractions " neutralised" and the 100 complete doses. Another portion will be due to the magnification of the experimental error in the determination of the lethal dose in the same way as an error of more than one whole dose arose in the corrosive sublimate experiments. If these two factors of themselves are not sufficient to account for the whole difference, then, apart from other considerations, it is probable that where bodies of high molecular weight are reacting the process only advances to a certain limit when the products of the reaction cause it to cease. This is, however, a hypothetical consideration.

6 Various observers have drawn attention to the following. When 100 times the quantity of antitoxin required to neutralise the lethal dose reacts with a multiple of the latter the animals experimented on, though they may escape immediate death, frequently suffer from, or even ultimately die with, chronic paralytic symptoms. Naturally the hypothetical non-toxic bodies, which have been held to be respon sible for the incapacity of 100 equivalents of antitoxic serum to account for 100 lethal doses of toxin bouillon, have been designated as the cause of this paralysis It will be noted that bodies previously termed non-toxic (they are defined as inert but chemically active) have, to suit this interpretation, to become toxic and paralysis-producing bodies. In determining the absolute minimal lethal dose of diph theria toxin bouillon the acutely fatal doses are succeeded by others which kill after long intervals up to three or four weeks. Among the guinea-pigs which die after three weeks there are occasionally some which die after the manifesta. tion of paralysis. Some which manifest paralysis recover. I have made no special observations into this matter with toxin bouillon. The above observations have been made incidentally at various times. With a diphtheria toxin precipitated by means of sulphate of ammonium, however, I have been able to confirm the observations of Ransom in regard to the paralysing action of various fractions of the lethal dose of such a toxin. This simply means that doses of toxin less than the acutely lethal dose may produce paralysis, may indeed produce paralysis and death subacutely. It does not much matter whether the toxin used was contained in fluid diphtheria toxin bouillon, or in precipitated and dried diphtheria toxin bouillon. Some may, howerer, object that the precipitation of the toxin might modify it and therefore be responsible for the manufacture of a product able to bring about paralysis. Personally
I do not entertain the validity of this objection. When these phenomena can be called forth by the toxic body itself, without the addition of antitoxin, I must confess that I do not see any necessity for assuming that the causative agent producing the same phenomena in the cases of doses of toxin imperfectly satisfied by antitoxin is due to a factor other than the toxin present in the bouillon. If the product of the reaction between toxin and antitoxin be able to react with a further addition of either of the latter, it may be that toxin partially chemically neutralised but not saturated with antitoxin gives rise to a product not indifferent to the organism. It would be speculation to ascribe the paralytic phenomena to the activity of this prodnct when toxin alone can produce them. It seems to me that the facts ascertained, however, only justify us in concluding that by means of antitoxin the acutely lethal dose is reduced in its toxicity so that it ceases to be acutely fatal but remains sufficiently toxic to call forth the same paralytic symptoms as the before-mentioned fraction of the lethal dose and for the same reasons. If not, then one is forced to the assumption that the fraction of the mere lethal dose which calls forth paralysis is able to effect the same degree of injury as can be effected by the sum-total of the difference between 100 and the actual number of doses which are rendered physiologically inert by 100 equivalents of antitoxin This last seems a needless assumption. ${ }^{B}$ I believe these paralytic symptoms were earliest described by Woodhead and Madsen at a date when the present widely disseminated hypothesis of immunity had not been enunciated, and it is interesting to observe th at Madsen in describing the occurrence of paralysis in guinea-pigs which had received multiple doses of toxin imperfectly neutralised physiologically also accompanies his excellent description of this paralysis in guinea-pigs by an equally detailed description of paralytic phenomena noticed in a horse. In the case of the guinea-pigs the paralysis was induced by doses of toxin imperfectly " neutralised" by antitoxin; in the case of the horse, however, it was brought about by repeated injections of sublethal doses ${ }^{7}$ of toxin alone. The toxin used by Madsen appears to have been toxin bouillon and not precipitated toxin.

It may be asked, Why is it that if sub-lethal doses of toxin alone produce paralysis this phenomena is so rarely seen in determinations of the lethal dose and so often found in experiments directed to determining the physiological neutralisation power of 100 equivalents of antitoxin? The answer is that in the latter case the doses are grouped in a manner which tends to bring into prominence the zone intervening between acutely lethal and non-lethal doses. In the case of the determination of the lethal dose the point to which attention is directed is at the other end of the scale, is the letbal issue, and nowadays a lethal issue within four days. If the zone within which such a pronounced effect as death occurs varies within very considerable limits it is extremely likely that the zone within which an effect manifesting itself after three or four weeks in such very delicate and varying degrees as the diphtheritic paralysis does will vary within wider limits, and guinea-pigs are not ideal animals in which to observe paralytic phenomena. Attention has been directed to the causation of death and the less pronounced manifestations at the other end of the scale of active doses have been very rarely studied.

The considerations contained in the preceding paragraphs show that it is not correct to require that if a certain quantity of antitoxin renders non-lethal a single fatal dose of toxin the hundredfold multiple of this quantity should render physiologically inert 100 similar fatal doses. In taking a large multiple of the fatal dose the fractions remaining potent are multiplied also. They become an agency which has to be reckoned with and may even become a multiple of the toxic power of the fatal dose itself. In cases where their effects become multiples of that of the fatal dose they are largely, if not entirely,

6 Put otherwise, the relatively non-toxic modifications of toxin (toxoids, toxones, \&c.) assumed to account for the paralytic symptoms of mixtures of toxin and antitoxin are unique among agents acting deleteriously on the animal body in that the severity of their action does not vary directly with the size of the dose, the same result being obtained by the fraction contained in the sub-lethal dose of toxin bouillon and by, e g., more than 100 times that quantity when the action of the ordinary toxin is eliminated by addition of antitoxin

7 One is forcibly reminded of the relation of peripheral paralysis due to alcohol or arsenic to the acute poisoning by these agents. 
responsible for the difference between 100 and the total number of fatal doses accounted for by 100 equivalents of antitoxin. Where they are augmented into sub-lethal effects they may become responsible for the occurrence of paralysis. This line of investigation of itself affords therefore absolutely no evidence of the presence in diphtheria toxin bouillon either of non-toxic modifications of diphtheria toxin which are still able to neutralise antitoxin, or of modifications of diphtheria toxin having the specific power of producing paralytic phenomena.

PART II.-SOME THEORETICAM CONCLUSTONS DRAWN FROM the Phenumena Manifested when more Toxis is ADDED TO WHAT HAS BEEN DETERMINED TO BE A Physiologically Neutral Mixture of Toxin and ANTITOXIN.

The physiologically neutral mixture of toxin and antitoxin may be composed of any multiple of the lethal dose and the equivalent quantity of antitoxic serum. It has, however, become customary to take as a basis the hundredfold multiple of the lethal dose or, more correctly, to take what is regarded as being the hundredfold multiple of the quantity of antitoxin necessary to abolish the action of the simple lethal dose. The hundredfold multiples have been chosen by distinguished observers for the purpose of instituting a comparison with the phenomena and conclusions drawn from the abolition of the fatal action of a lethal dose of toxin by antitoxin and the absence of this abolition if the hundredfold multiples be taken. No attempt has been made by those who have made this comparison to show that it is a legitimate one. The following considerations will show that it is not. An explanation common to the two sets of phenomena compared in this way has been given on the assumption that they must be due to identical factors. It has been assumed that these phenomena are due to the different affinities for antitoxin of non-toxic or relatively non-toxic modifications of toxin. I have concluded that the line of investigation considered in Part $\mathrm{I}$. gives no evidence of the existence of such bodies. The two phenomena com. pared are, however, not comparable in this way, for it will be shown that they are not due to identical factors but stand in no direct relation to one another. Some observations which $I$ have made with diphtheria toxin and antitoxin in vivo and with ricin and its antitoxin in vitro have given distinct evidence that either (1) the proportion of antitoxin necessary to effect abolition of the action of a definite quantity of toxin is quantitatively in excess of the portion of toxin actually chemically neutralised, or (2) the product of the reaction between toxin and antitoxin is capable ultimately of modifying the action of higher amounts of toxin than the quantity with which the antitoxin reacts in the first instance.

In illustration of what has just been said the following experiments with ricin and its antitoxin (antiricin) may be given.

Ricin-Abolition of the Action of the Unit Clumping Dose means Interference with the Aotion of Higher Doses.

(a) Determination of the unit cly:mping dose of ricin (solution $=1$ in 10,000 of 0.86 per cent. saline solution).

\begin{tabular}{|c|c|c|c|c|}
\hline $\begin{array}{c}\text { Solution of ricin } \\
1 \text { in } 10,000 .\end{array}$ & & $\begin{array}{l}\text { cent. } \\
\text { spensio }\end{array}$ & & $\begin{array}{c}\text { Result after two hours at } \\
38^{\circ} \mathrm{C} \text {. }\end{array}$ \\
\hline 1.0 c.c. & + & 1 c.c. & ...... & Complete clumping. \\
\hline 0.75. & + & , & ....... & ," \\
\hline 0.50, & + & ," & ....... & ," \\
\hline 0.35, & + &, & ....... & Almost, but not complete \\
\hline 0.25, & + & ," & ...... & ", \\
\hline $0 \cdot 15$ & + & , & ... & ," \\
\hline
\end{tabular}

Conclusion. -0.5 cubic centimetre of 1 in 10,000 ricin clumps completely 1 cubic centimetre of the blood suspension.

(b) Determination of dose of serum necessary to abolish the action of the above dose of ricin.

\begin{tabular}{|c|c|c|c|c|c|}
\hline \multicolumn{2}{|c|}{$\underset{1 \text { in } 10,000}{\text { Ricin }}$} & \multicolumn{2}{|l|}{$\begin{array}{l}\text { Serum } \\
1 \text { in } 20 .\end{array}$} & \multicolumn{2}{|c|}{$\begin{array}{l}\text { Result after two } \\
\text { hours at } 38^{\circ} \mathrm{C} .\end{array}$} \\
\hline 0.5 c.c. & + & 0.20 e.c. & & Some clur & ping. \\
\hline ", & + & 0.25, & $\begin{array}{l}\text { Remained } \\
10 \text { minutes }\end{array}$ & Trace of & ", \\
\hline " & + & 0.30, & at $38^{\circ} \mathrm{O}$ & No & \\
\hline ," & + & & then blood & ", & " \\
\hline ," & + & 0.40, & added as & " & " \\
\hline ", & & 0.50, & & & ", \\
\hline
\end{tabular}

Conclusion. -0.3 cubic centimetre of serum dilution abolishes the action of the unit dose of ricin. In order to obviate the pseudoantitoxic action of normal serum the serum was diluted 1 in 20 . (c) Determination of the limits of action of 0.3 cubic centimetre of the serum dilution.

\begin{tabular}{|c|c|c|c|c|c|}
\hline \multicolumn{2}{|c|}{$\underset{1 \text { in } 10,000 .}{\text { Ricin }}$} & \multicolumn{2}{|r|}{$\begin{array}{l}\text { Serum } \\
1 \text { in } 10 .\end{array}$} & \multicolumn{2}{|r|}{$\begin{array}{l}\text { Result after two } \\
\text { hours at } 38^{\circ} \mathrm{C} \text {. }\end{array}$} \\
\hline 0.50 & c.c. & + & 0.3 c.c. & \multirow{11}{*}{$\begin{array}{l}\text { Remained } \\
10 \text { minutes } \\
\text { at } 38^{\circ} \mathrm{C} ., \\
\text { then blood } \\
\text { added as } \\
\text { above. }\end{array}$} & No clumping. \\
\hline 0.55 & $"$, & + & " & & Slight clumping. \\
\hline 0.60 & $"$ & + & , & & \\
\hline 0.65 & , & + & , & & \\
\hline $0 \cdot 70$ & $"$ & + & $n$ & & \\
\hline $0 \cdot 75$ & ", & + & , & & \\
\hline $0 \cdot 80$ & ", & + & , & & $\begin{array}{l}\text { Gradually increasin } \\
\text { clumping. }\end{array}$ \\
\hline $0 \cdot 85$ & $"$, & + & ," & & \\
\hline 0.90 & $"$ & + & ", & & \\
\hline $1 \cdot 00$ & $"$ & + & $"$ & & \\
\hline $1 \cdot 05$ & , & + & , & & Dnly moderate clumpi \\
\hline \multicolumn{2}{|c|}{$\begin{array}{l}\text { Control } \\
\text { ricin. }\end{array}$} & & $\begin{array}{l}\text { Serum } \\
1 \text { in } 10 .\end{array}$ & & \\
\hline \multicolumn{2}{|c|}{0.5 c.c. } & + & 0.3 c.c. & & No clumping. \\
\hline
\end{tabular}

(d) Confirmation of experiment (c).-The conditions were the same as in $(c)$ but here quantities of saline solution ( $(0.86$ per cent.) were added to each test-tube to raise the bulk to one cubic centimetre before adding the serum; this allows of stating the result in absolute quantities of ricin contained in one cubic centimetre in each tube.

\begin{tabular}{|c|c|c|c|c|c|}
\hline \multicolumn{2}{|c|}{$\begin{array}{l}\text { Ricin } \\
1 \text { in } 5000 .\end{array}$} & & $\begin{array}{l}\text { Serum } \\
1 \text { in } 10 .\end{array}$ & & $\begin{array}{l}\text { Result after two } \\
\text { hours at } 38^{\circ} \mathrm{C} .\end{array}$ \\
\hline & & + & 0.3 c.c. & & No clumping. \\
\hline 0.30 &, & + &, & & (P) Slight clumping. \\
\hline 0.35 & , & + &, & & \\
\hline 0.40 & , & + & , & & \\
\hline 0.45 & , & + & , & & \\
\hline 0.50 &, & + &, & Remainer & \\
\hline 0.55 & $"$, & + & , & $\begin{array}{l}10 \text { minutes } \\
\text { in incubator }\end{array}$ & \\
\hline 0.60 & , & + & , & at $38^{\circ} \mathrm{C}$ & Gradually increasing \\
\hline 0.65 &,, & + & "' & then blood & clumping. \\
\hline $0 \cdot 70$ & , & + & , & added as & \\
\hline 0.75 & $"$, & + & , & & \\
\hline 0.80 & ", & + & ," & & \\
\hline 0.85 & , & + & , & & \\
\hline 0.90 & ", & + & $"$ & & \\
\hline
\end{tabular}

Very strong but not com

Control

ricin.

0.25 c.c. + Saline + blood

$0.25, \quad+\quad$ Serum 1 in 10

0.3 c.c. + blood.

plete clumping.

Complete clumping.

No clumping.

Conclusion from experiments $(c)$ and $(d)$ : The quantity of serum which abolishes the action of 0.05 milligramme of ricin also interferes with the action of much higher doses of ricin.

The experiments were performed with the red blood corpuscles of the rabbit after centrifugalising free from serum and suspending them in 086 per cent. saline solution. The antitoxic serum was also derived from a rabbit. As is well known, ricin clumps together the red blood corpuscles of the rabbit. In the first instance one determined the smallest dose of ricin able completely to clump one cubic centimetre of the blood suspension after two hours' contact in the incubator at $38^{\circ} \mathrm{C}$. Then in another series to it varying quantities of the antitoxic serum were added to this dose of ricin as a constant quantity. After the serum and the ricin had remained in contact for ten minutes in the incubator ${ }^{8}$ the blood was added and after two hours in the incubator the degree of clumping was noted. In this way the smallest dose of antitoxin able to abolish the clumping action of a dose of ricin taken as unity was determined. In two other series the quantity of antitoxic serum thus determined remained constant; the quantities of ricin varied. The results showed that the smallest quantity of antitoxin (antiricin) able to effect abolition of the action of the dose of ricin taken as unity was also able to modify the effect of much larger doses of ricin when the experimental conditions were altered. For in the two series with varying doses of ricin abolition of the action of the dose of ricin taken as unity also involved an extension of the abolition much further up the series of doses of ricin. Where one would have expected the unit dose of ricin to be again "free" to act-e.g., at two or three times unity-the action manifested fell much behind that of the unit dose of ricin. From this experiment, which has been confirmed by many repetitions, I draw the conclusion that either (1) one must make the assumption that the proportion of antitoxin necessary to effect abolition of the action of a definite quantity of ricin is quantitatively in excess of the proportion of ricin actually physiologically neutralised, and of this

8 This duration was chosen to obviate fallacies arising in precipital tion of the constituents of the ricin solution by the antiricin serum. 
there is no evidence ; or (2) that the product of the reaction with the smaller quantity of ricin is capable ultimately of taking up a higher amount of ricin than that quantity with which the antiricin reacts chemically in the first instance and that neither in the case of the smaller nor of the larger doses of ricin within a definite range of doses is there free ricin or free antiricin.

In the above experiment the toxin was ricin and the experiment was performed in vitro. In the case of diphtheria and tetanus toxins and antitoxins I have met with phenomena of a similar nature and such as have been recorded especially by Knorr and by those who have advanced the views under criticism. When more toxin i added to what has been determined to be a physiologically neutral mixture of toxin and antitoxin it is neces-ary in order to obtain again the full action of the dose of toxin taken as unity to add, not one unit of toxin, but several ; the reasons are possibly identical with those obtaining in the above experiment with ricin. In the case of ricin the full effect of the dose of ricin taken as unity was in the presence of antiricin not obtained again till more than three times this unit was present. In the case of the experiments with diphtberia toxin and antitoxin a physiologically neutral mixture (made on the basis of hundredfold multiples) was not converted into a mixture able to produce again the effect of the dose taken as unity till more than three times the latter quantity of toxin had been added. This was the smallest quantity which caused the full effect to be reproduced and obviously the considerations in the first part of this paper will have made it evident that the quantity required to produce this effect will vary in proportion to the amount of unavoidable error in determining what was a physiologically neutral mixture. It has been demonstrated in Part I. that what is defined as a physiologically neutral mixture is no constant quantity but that by this term a whole series of mixtures (in which at one end there is present a qualitative excess of antitoxin and at the other end just enough antitoxin) are equally well designated and, indeed, are indistinguishable by any methods at our command. These experiments lead to the natural conclusion that a constant volume of a solution of antitoxin reacts in more than one quantitative relation with volumes of toxin as illustrated here, especially for ricin.

No further explanation is needed in order to point out the absolute difference and entire independence of the phenomena which are responsible on the one hand for the abolition of the fatal action of a lethal dose of toxin by antitoxin and the absence of this abolition if the hundredfold multiples be taken; and, on the other hand, the necessity for adding more than one unit of toxin to what has been determined to be a physiologically neutral mixture of toxin and antitoxin in order that the full effect of a unit of toxin may be again manifested. It will now be evident that any attempt to explain these distinct phenomena by making them dependent upon the same causative factors must be wrong. In particular the view that the factors in both instances are non-toxic or relatively non toxic modifications of toxin of the same, a greater, or a less affinity for antitoxin than is characteristic of the toxin, is not in accordance with the facts. The assumption that the existence of physiologically inert but chemically active modifications of toxin causes the apparent non-fulfilment of the law of multiples must be given up, although it is hypothetically adequate to account for the phenomenon, and the explanation sought in the unavoidable experimental errors of a qualitative and quantitative nature and in toxin and antitoxin reacting with one another in more than one fixed proportion.

\section{CONCLUSTONS.}

In investigating toxin alone it has been shown that-

1. The volume containing the lethal dose can be converted into one no longer lethal within the time limit by diminishing the total volume by a small but measurable fraction of the whole.

2. A measurable quantity of toxin solution can be borne without the production of any symptoms.

Both these effects can be obtained in a different guise when one is dealing with a mixture of toxin and antitoxin, for it has been ascertained that-

3. The poisonous action of the lethal dose may be abolished within the time limit by the addition of a very small quantity of antitoxin; the experiment gives evidence of a qualitative reduction in the toxicity itself, but no evidence of the occurrence of the quantitative reduction referred to in conclusion 1.
4. When the toxic power of a quantity of toxin has been reduced by antitoxin to such an extent that no symptoms appear a measurable degree of toxic power is still potent qualitatively, but we are not entitled to state this in the quantitative terms of conclusion 2.

It has further been found that-

5. The lethal dose is separated from the non-lethal not by a hard-and-fast line but by a zone wherein are contained appreciable fractions of the lethal dose; the data here are quantitative.

6. In mixtures of toxin and antitoxin in such proportions that a lethal issue occurs and does not occur, the margin between the toxic powers of such mixture is not sharp but is a zone, and the actions standing at the upper and lower limits of this zore differ from each other by a measurable fraction of the total toxicity; the data here are purely qualitative.

7. It is not justifiable to assume $a$ priori that one volume of antitoxin solution always fully accounts for one volume of toxin solution when mixed in varying multiples. This pre. supposes that quantitative excess of either is possible and measurable, and ignores the fact that we cannot estimate the quantitative and chemical relations of toxin and antitoxin, but merely the qualitative physiological characters of the mixture, and this only when the toxic power present is manifestly in excess. It is preferable to speak of the retention of active toxicity or of active antitoxicity in characterising such mixtures. The phrases "free toxin" and "free antitoxin" may not be in accordance with the facts and should be abandoned.

8. The abuve facts indicate the limitations within which it is possible to determine the volume of the lethal dose of toxin and the volume of antitoxic serum sufficient to abolish its lethal action-i.e., the qualitative determination of a physiologically neutral mixture of smallest bulk. If the volumes thus determined be merely multiplied one hundredfold a qualitative indication of the amount of the experimental error present in the original determination is obtained, but no basis for deductions as to the quantitative and chemical relations existing between the toxin and antitoxin contained in the reacting volumes. The assumption that data thus obtained permit of conclusions of a far-reaching nature as to the quantitative relations and intimate chemical nature of the reaction involves a confounding of the abstract with the concrete.

9. The smallest quantity of antiricin which just suffices to abolish the action of a unit dose of ricin, that is the smallest qualitative equivalent of the latter, modifies the action of bigher doses of ricin. This shows that the unit dose of ricin is but one of a series of doses to which this quantity of antiricin has a qualitative relation and the fact can only be interpreted as proving that one and the same volume of antiricin has quantitative relations with more than one volume of ricin. At one end of the scale of doses of ricin, with which qualitative relations exist, there will be active if modified antitoxicity, as, e.g., with the unit dose of ricin, and at the other end active if modified toxicity, but neither ricin nor antiricin is really "free." The phenomena noted for mixtures of diphtheria toxin and antitoxin and for tetanus toxin and antitoxin are exactly comparable to those obtained with ricin and antiricin and are to be explained by the existence between these toxins and antitnxins of similar quantitative relations in accordance with which they react with one another in more than one fixed proportion.

The results on which this paper is based were obtained previously to November, 1902, and have already been communicated to the scientific assessors of the Worshipinul Company of Grocers; other duties have interfered with earlier publication. In a recent number of the Annales de l'Institut Pasteur Bordet has announced similar results. Bordet followed different lines of investigation and his results were of a nature to warrant him drawing more definite and far-reaching conclusions than $I$ feel justified in making at present.

Harley House, W.

Royal Devon and Exeter Hospital.-In accordance with ancient custom the committee and governors of this hospital, accompanied by the mayor and corporation and representatives of other public bodies, will go in procession on Sunday afternoon, Oct. 18th, to Exeter Cathedral where a sermon will be preached by the Rev. Chancellor Edmonds and a collection will be made on behalf of the funds of the institution. 